Journal of Statistical Physics, Vol. 93, Nos. 1/2, 1998

\title{
Graphical Representations for Ising Systems in External Fields
}

\author{
L. Chayes, ${ }^{1}$ J. Machta, ${ }^{2}$ and O. Redner ${ }^{3}$
}

Received May 8, 1998; final June 26, 1998

A graphical representation based on duplication is developed that is suitable for the study of lsing systems in external fields. Two independent replicas of the Ising system in the same field are treated as a single four-state (Ashkin-Teller) model. Bonds in the graphical representation connect the Ashkin-Teller spins. For ferromagnetic systems it is proved that ordering is characterized by percolation in this representation. The representation leads immediately to cluster algorithms; some applications along these lines are discussed.

KEY WORDS: Cluster methods; external fields; RFIM.

\section{INTRODUCTION}

Graphical representations have provided a powerful tool for both the analytical and numerical study of statistical mechanics systems. However, the full power of these methods can only be realized when the phases and the phase transitions in the original system are easily identifiable in the graphical representation. Usually this involves the percolation properties of the graphical representation. Consequently, the subject is still something of an "art"; various systems are treated on a case-by-case basis. Despite the recent progress on systems other than Potts models (cf. [CM II] and references therein) an apparent requirement is a high degree of internal symmetry for the individual degrees of freedom.

${ }^{1}$ Department of Mathematics, University of California, Los Angeles, California 90095-1555; e-mail: lchayes (a math.ucla.edu.

${ }^{2}$ Department of Physics and Astronomy, University of Massachusetts, Amherst, Massachusetts 01003-3720; e-mail: machta $\alpha$ phast.umass.edu.

${ }^{3}$ Institut für Theoretische Physik, Universität Tübingen, D-72076 Tübingen, Germany; e-mail: oliver.redner $(a$ student.uni-tuebingen.de. 
In this paper, we will treat the simplest possible system with no internal symmetry, namely the ferromagnetic Ising model in an external field. The representation is actually that of a duplicated (or "replicated") system and is closely related to the so called red/blue representation of the Ashkin-Teller model that was introduced in [CM I]. Before launching into a full description of this representation - the subject of the next section--let us illustrate the problems that beset the standard approaches.

Consider the usual Ising Hamiltonian:

$$
\mathscr{H}=-\sum_{\langle i, j\rangle} J_{i, j} \sigma_{i} \sigma_{j}-\sum_{i} h_{i} \sigma_{i}
$$

where the bonds $(\langle i, j\rangle)$ and sites (i) are part of some regular graph, the $J_{i, j}$ are positive and $-\infty<h_{i}<+\infty$. To simplify a number of technical points, it will be assumed that the model is short ranged, that is to say, the number of bonds emanating from any site is uniformly bounded.

In case $h_{i} \equiv 0$, there is the standard random cluster representation [FK] for bond configurations $\omega$ : In finite volume let

$$
W(\omega)=B_{J}(\omega) 2^{c(\omega)}
$$

where $B_{\mathbf{J}}$ is the Bernoulli factor of $1-e^{-2 \beta J_{i, j}}$ for each occupied bond of $\omega$ and $e^{-2 \beta J_{l, j}}$ for each vacant bond and $c(\omega)$ is the number of components. (Here we ignore modifications due to boundary conditions.) Since, for each $\omega$, these quantities (weights) are non-negative they define a probability measure on the collection of all bond configurations. As such, these measures admit infinite volume limits. It is remarked that for regular infinite volume systems, e.g., nearest neighbor models on $\mathbb{Z}^{d}$, the percolation of these bonds corresponds to positive spontaneous magnetization in the spin-system [CK, ACCN].

For $h_{i}$ not identically zero, there are two obvious approaches both of which lead to unsatisfactory results: First we may include the magnetic fields directly in the formula for the weights. The result is

$$
W(\omega)=B_{\mathbf{J}}(\omega) \prod_{\alpha=1}^{c(\omega)} 2 \cosh \left[\mathbf{h}\left(K_{\alpha}\right)\right]
$$

where the product runs over the clusters $K_{1}, \ldots, K_{c(\omega)}$ and $\mathbf{h}\left(K_{\alpha}\right)=$ $\beta \sum_{i \in K_{\alpha}} h_{i}$. In other words each cluster is given a weight in accord with the field energy obtained if all spins of the cluster are of the same sign. The second approach-which is most useful if all the $\left(h_{i}\right)$ are of the same sign-is the introduction of ghost bonds. In general, the ghost system is defined as follows: clusters are defined and preliminarily weighted as in the 
zero field case. In addition, for every site $i$ such that $h_{i}>0$, there may be an "up" ghost bond which carries the weight $1-e^{-2 \beta h_{t}}$ or no such bond with weight $e^{-2 \beta h_{i}}$. Similarly, if $h_{j}<0$, the site $j$ may get a "down" ghost bond with weight $1-e^{-2 \beta\left|h_{j}\right|}$, etc. However, the system is constrained so that no cluster may have ghost bonds of both types. Thus, if $\bar{\omega}$ denotes the extended system of ordinary and ghost bonds the weight for $\bar{\omega}$ is given by

$$
\bar{W}(\bar{\omega})=\Phi(\bar{\omega}) B_{\mathbf{J}, \mathbf{h}}(\bar{\omega}) \prod_{\alpha=1}^{c(\omega)} v_{\alpha}
$$

here $\Phi(\bar{\omega})$ is the function that is one if the above mentioned constraint is satisfied and zero otherwise, $v_{\alpha}$ is 2 if there are no ghost bonds in the $\alpha$ th cluster and 1 otherwise and $B_{\mathbf{J}, \mathbf{h}}(\bar{\omega})$ is the Bernoulli weight for the configuration $\bar{\omega}: 1-e^{2 \beta J_{i, j}}\left(e^{2 \beta J_{i, j}}\right)$ if the bond $\langle i, j\rangle$ is occupied (unoccupied) and $1-e^{2 \beta\left|h_{i}\right|}\left(e^{2 \beta\left|h_{i}\right|}\right)$ if the ghost bond at $i$ is occupied (unoccupied). It is not hard to show that the weights in Eq. (3) are obtained from those in Eq. (4) by summing over the ghost bonds.

The difficulty with either of these representations is manifestly apparent. Consider the case $h_{i} \equiv h>0$ on the usual $d$-dimensional lattice with $d \geqslant 2$. By the Lee-Yang theorem, there is no phase transition as temperature is varied. Nevertheless, it is clear that in the graphical representation, there is a percolation transition at some finite $\beta_{f}(h) \leqslant \beta_{c}$. Thus, these representations are susceptible to false percolation transitions. In less trivial situations such as random field problems, it can be argued that such false percolations will also occur. Cluster algorithms based on this representation are ineffective because of the weighting of cluster flips and the problem of false percolation: Notwithstanding the fact that large-scale clusters may be of no particular interest, they tend to become dynamically frozen. For example, in the second representation, no cluster can flip unless it is devoid of ghost bonds which occurs very rarely for large clusters. These effects lead to severe slowing down both for critical and non-critical values of the parameters.

Attempts have been made to circumvent these difficulties by restricting the size of clusters (e.g., [NB]) - with some degree of success-but in principal, there can be no percolative signal of the phase transition in any such representation.

It is therefore of interest to produce representations for spin-systems in arbitrary external fields; the subject of this work. In this regard, we have recently learned of a related approach to these sets of problems that is applicable to systems with periodically varying fields. These methods are described in $[\mathrm{HBb}]$ and $[\mathrm{HBc}]$ and were applied to the hard-core lattice gas (a limiting case of the staggered field problem) $[\mathrm{HBa},[\mathrm{HBb}]$ and 
related systems [ $\mathrm{HBc}]$. We shall describe the similarities (and differences) between this method and the current method in the conclusions section. At present, these approaches are, by and large, restricted to Ising-type systems. Nevertheless, this covers a number of problems that are of current interest such as the random-field Ising model. In the context of the staggered-field Ising system, an algorithm based on this representation has already been tested [RMC] with quite satisfactory results.

\section{RED/BLUE REPRESENTATION IN ZERO FIELD}

Let us start with the zero field case. Much of this material appears in [CM I] (Appendix B3) in the context of the Ashkin-Teller model. Consider the duplicated system so that at each site $i$, we have $\sigma_{i}= \pm 1, \tau_{i}= \pm 1$. We will envision the duplicated problem as a 4-state spin model, in particular, the 4-state clock model. Let us use $s_{i}$ to denote the spin variable, regarded as one of four points on the circle and $\underline{s}$ as notation for a spin configuration. If $\langle i, j\rangle$ is a bond of the lattice, there are three possible energy states for the spins $s_{i}$ and $s_{j}$, the highest of which is set to zero without loss of generality. Thus we define $\mathscr{E}_{i, j}^{0}=4 J_{i, j}$ and $\mathscr{E}_{i, j}^{1}=2 J_{i, j}$. The Hamiltonian for the duplicated system is given by

$$
H(\underline{s})=-\sum_{\langle i, j\rangle}\left[\mathscr{E}_{i, j}^{0} \delta_{s_{i}=s_{j}}+\mathscr{E}_{i, j}^{1} \delta_{s_{i}=s_{j}+1}+\mathscr{E}_{i, j}^{1} \delta_{s_{i}=s_{j}-1}\right]
$$

where the $\delta$ 's are one if the indicated condition is satisfied and vanish otherwise and we have used additive notation for the group structure of the spin-states. Explicitly, $s \rightarrow s+1$ means $(++) \rightarrow(+-) \rightarrow(--) \rightarrow$ $(-+) \rightarrow(++) .^{4}$

For each $\langle i, j\rangle$ we may expand the statistical weight associated with $H$ as:

$$
\begin{aligned}
& e^{\beta \mathscr{E}_{i, j}^{0} \delta_{s_{l}}=s_{j}} e^{\beta \mathscr{E}_{i, j}^{1} \delta_{s_{i}}=s_{j}+1} e^{\beta \delta_{i, j}^{1} \delta_{s_{l}=s_{j}-1}} \\
& =1+B_{i, j} \delta_{s_{i}=s_{j}}+R_{i, j} \delta_{s_{i}=s_{j}+1}+R_{i, j} \delta_{s_{i}=s_{j}-1}
\end{aligned}
$$

with $B_{i, j}=e^{\beta \mathscr{E}_{i, j}^{0}}-1$ and $R_{i, j}=e^{\beta \mathscr{E}_{i, j}^{1}}-1$. Note that no terms involving products of $\delta$ 's appear since the conditions are exclusive. Thus, there are four possibilities for each $\langle i, j\rangle:$ A "vacant" bond corresponding to the 1 , a "blue" bond corresponding to the term $B_{i, j} \delta_{s_{i}=s_{j}}$ and one of two types of "red" bonds which forces $s_{i}=s_{j} \pm 1$.

\footnotetext{
${ }^{4}$ We remark that the Hamiltonian in Eq. (5) differs from (two copies of) that in Eq. (5)) with $h_{i} \equiv 0$ - by an overall constant.
} 
A graphical configuration $\eta$ is obtained by a selection of one of the above four terms for each bond of the lattice. The weight of $\eta$ is given by

$$
\underline{V}(\underline{\eta})=\sum_{\underline{s}} \prod_{\langle i, j\rangle} \mathscr{R}_{\langle i, j\rangle}(\underline{\eta}) \delta_{\langle i, j\rangle}(\underline{\eta}, \underline{s})
$$

where $\mathscr{R}_{\langle i, j\rangle}(\eta)=1, B_{i, j}$ or $R_{i, j}$ according to whether a vacant, blue or red bond has been selected and $\delta_{\langle i, j\rangle}(\underline{\eta}, \underline{s})$ is the corresponding delta factor (or 1 for the vacant bonds).

These weights are not particularly difficult to evaluate explicitly nor are the results particularly illuminating. Of greater interest is the weight associated with all configurations $\eta$ with the same colored bonds but different types of red bonds. We will denote these equivalence classes by an unadorned $\eta$ and define $V(\eta)=\sum_{\eta \in \eta} \underline{V}(\eta)$. An explicit formula for $V(\eta)$ is also possible which we will give after the relevant notation is developed.

First it is noted that not all $\eta$ 's have non-zero weight. In particular, it is necessary and sufficient that each closed loop of non-vacant (hereafter "occupied") bonds of $\eta$ must consist of an even number of red bonds. Let $D(\eta)$ be the indicator that the above condition is satisfied. Next, let $F(\eta)$ denote the "bond fugacity" prefactor (which is the same for all $\eta$ 's in the equivalence class)

$$
F(\eta)=\prod_{\langle i, j\rangle} \mathscr{R}_{\langle i, j\rangle}(\eta)
$$

Finally, the geometric factors: It is observed that there are two relevant notions of connectedness in a configuration $\eta$, namely connected by blue bonds or connected by red and blue bonds, which we will call a grey connection. Let $C_{b}(\eta)$ denote the number of blue components in the configuration $\eta$ and $K(\eta)$ the number of components by the less stringent (grey) definition. Thus $K(\eta) \leqslant C_{b}(\eta)$; for example, if each bond is occupied and red, $K(\eta)=1$ while $C_{b}(\eta)$ is the total number of sites.

With the above in hand, the result is

$$
V(\eta)=F(\eta) D(\eta) 2^{K(\eta)} 2^{C_{b}(\eta)}
$$

We remark that we have not yet discussed boundary conditions; the above is strictly true only for free boundary conditions on an arbitrary (but finite) graph. We denote the associated measure by $v(-)$ with various super- and sub-scripted adornments to be appended as needed to indicate the lattice, the boundary conditions and the fields.

We introduce another measure, called the Edwards-Sokal measure, for the representation. This is a joint measure on bond and spin configurations 
that assigns an equal weight to each spin configuration that is "consistent" with the given bond configuration:

$$
V^{E S}(\underline{s}, \eta)=F(\eta) \Delta(\underline{s}, \eta)
$$

In the above, $\Delta(\underline{s}, \eta)$ vanishes unless (a) the spin values are constant in each blue component of $\eta$ and (b) the spin values $s_{i}$ and $s_{j}$ across each red bond of $\eta$ differ by one: $s_{i}=s_{j} \pm 1$. If (a) and (b) are both satisfied then $\Delta(\underline{s}, \eta)=1$. The Edwards-Sokal measures defined by these weights will be denoted by $v^{E S}(-)$. It is not difficult to see that

$$
\sum_{\eta} V^{E S}(\underline{s}, \eta)=e^{-\beta H(\underline{s})}
$$

and

$$
\sum_{\underline{s}} V^{E S}(\underline{s}, \eta)=V(\eta)
$$

Thus the bond marginal of $v^{E S}(-)$ is the red/blue random cluster measure and the spin marginal is the Gibbs measure for the system. The Edwards-Sokal measure facilitates an interpretation of the bond configuration $\eta$. In particular, connected clusters of blue sites represent spins that are all in the same state, separate clusters not connected by any bonds are uncorrelated from one another and sites connected by an even (odd) number of red bonds represent spins that agree $\bmod 2$ (differ by \pm 1 ).

Boundary conditions are readily accounted for in the red/blue random cluster measures. If $A$ is the graph and $\partial A$ the boundary sites, a single spin specification on $\partial A$ divides $\partial A$ into (at most) four components. These components are treated as a single site in the counting of $K(\eta)$ and $C_{b}(\eta)$. Furthermore, there are restrictions on the configuration $\eta$, e.g., no two of the boundary components can be connected by blue bonds. Of particular interest are the wired boundary conditions where all the boundary sites are treated as a single component. In the absence of fields, the boundary type may be identified as any of the four states or superpositions thereof.

We now describe a cluster algorithm based on the red/blue representation. Starting from a spin configuration $\underline{s}$, each $\langle i, j\rangle$ is checked for the conditions $s_{i}=s_{j}$ or $s_{i}=s_{j} \pm 1$. If $s_{i}=s_{j}$, a blue bond is placed on $\langle i, j\rangle$ with probability $b_{i, j}=B_{i, j} /\left[1+B_{i, j}\right]=1-e^{-4 \beta J_{i, j}}$ or is declared vacant with probability $1-b_{i, j}$. If $s_{i}=s_{j} \pm 1$, a red bond is placed on $\langle i, j\rangle$ with probability $r_{i, j}=R_{i, j} /\left[1+R_{i, j}\right]=1-e^{-2 \beta J_{i, j}}$ or the bond is declared vacant with probability $1-r_{i, j}$. On all bonds for which $s_{i}$ and $s_{j}$ are completely opposed $\left(s_{i}=s_{j}+2\right.$, i.e., $\left.s_{i}=-s_{j}\right)$ the bond is always vacant. All bond moves are performed independently. This creates the configuration $\eta$. 
Given the configuration $\eta$, the updated spin configuration is created by two types of cluster moves. First, the spins in each isolated grey cluster can be coherently rotated by $0, \pm 1$, or 2 with equal probability. Second, the spin type of all spins in a blue cluster (including single spins with no blue bonds) can be reversed $(s \rightarrow-s)$ with probability $1 / 2$. We will refer to these as grey moves and blue moves respectively. The cluster moves are all performed independently. This algorithm is of the Swendsen-Wang [SW] type and it is straightforward to show, following [ES], that it simulates the Edward-Sokal measure of Eq. (10). For more details (and more formal descriptions) concerning general algorithms of this sort, the reader should consult $[\mathrm{CM}$ I], [CM II]

Remark. The strength of this representation is that percolation (essentially of blue bonds) can be directly related to the non-vanishing of the spontaneous magnetization. This will be discussed in full detail in the next section. The principal difficulty is the presence of large grey clusters which, under a variety of conditions, will hinder rapid equilibration. Indeed, at moderate temperatures above the magnetic ordering temperature, there is, generically, percolation of grey bonds. (For example, this can be established by rigorous bounds for the $2 d$ square lattice.) Furthermore at low temperatures, the rapid "tunneling" events characteristic of better representations are inhibited. For example consider a situation where $\beta \gg 1$ and suppose that all the spins on the left side of a box are in the state ++ while on the right side of the box all spins are +- . Here both the left and right sides will be tied up in a single cluster. If we use two independent SW algorithms (for the Ising case, $\mathscr{E}^{00}=2 \mathscr{E}^{1}$ or, in the general Ashkin-Teller case the bilayer representation) the two clusters are disjoint and the interface will disappear in a few Monte Carlo steps. However with the red/blue representation, there would nearly always be red bonds connecting the left and right regions which prevent full alignment of the box; in this case, an extremely long time is required for equilibration. This represents the principal reason that this approach was abandoned as a tool to study the Ashkin-Teller model. However, as we shall see, in the presence of external fields, the red/blue representation has significant advantages over the alternatives.

\section{RED/BLUE REPRESENTATION WITH FIELDS}

Before we actually introduce the fields, let us present one more refinement which, in the absence of fields is somewhat redundant. Consider a configuration $\eta$. Recall that each blue cluster of $\eta$ represents spins that are 
all in the same state. Now a blue cluster may be part of some larger grey cluster but the dynamics described in the previous section allows the spins of each blue cluster to flip ( $++\leftrightarrow--,+-\leftrightarrow-+)$ leaving the remaining spins of the grey cluster invariant. But then a further classification is allowed for the blue clusters namely whether the cluster is in the $(++,--)$ or $(-+,+-)$ states. In the former case, we will say that the cluster is dark-blue and in the latter, light-blue. As we shall see, percolation of the light-blue bonds represents the onset of magnetic ordering.

We will denote these red, dark-blue and light-blue (RDLB) configurations by $\Xi$ 's. It is seen that the weight of a configuration $\Xi$ is given by

$$
\mathbf{V}(\Xi)=F(\Xi) \mathbb{D}(\Xi) 2^{C_{b}(\Xi)}
$$

where $F(\Xi)$ has its previous meaning, $C_{b}(\Xi)$ is the number of blue clusters-both dark and light-and $\mathbb{D}(\Xi)$ indicates that within each grey cluster, all paths between pairs of light-blue clusters consist of an even number of red bonds, similarly for pairs of dark-blue clusters while any path between a dark-blue and a light-blue cluster consists of an odd number of red bonds. We note that the "grey moves" of the algorithm described in the previous section (where the spins of the grey clusters were coherently rotated) consists, with probability $1 / 2$, of exchanging dark-blue for light-blue within each grey cluster. As such it is easy to recover the weights in Eq. (9) from those of Eq. (12)-for each grey cluster, one sums over both these possibilities. We denote the measure defined by the weights in Eq. (12) by $\mathbb{V}(-)$ and the associated Edwards-Sokal measure by $\mathbb{V}^{E S}(-)$.

Finally we remark that the division of blue into dark and light degrees of freedom creates more candidates for the boundary conditions. In particular we must now separately consider light-wired boundary conditions corresponding, in the spin system, to +- or -+ boundary conditions or dark-wired corresponding to ++ or -- boundary conditions. We reiterate that in the absence of external fields, these are all equivalent: the graphical problem is invariant under the exchange of dark and light labels.

We are finally ready to introduce the fields. Consider the Ising Hamiltonian as given in Eq. (1). Upon duplication we have

$$
H=H(\underline{s})=\mathscr{H}(\underline{\sigma})+\mathscr{H}(\underline{\tau})=-\sum_{\langle i, j\rangle} J_{i, j}\left[\sigma_{i} \sigma_{j}+\tau_{i} \tau_{j}\right]-\sum_{i} h_{i}\left[\sigma_{i}+\tau_{i}\right]
$$

The result is clear: the fields do not act on the spins $s_{i}$ that are in the states $(+-)$ or $(-+)$ and hence on the light-blue clusters. In the construction of the graphical representation, we may either go the route of ghost bonds 
(as in Eq. (4)) or field energies (as in Eq. (5)). The latter choice is somewhat easier and gives us the weights

$$
\mathbf{V}(\Xi)=F(\Xi) \mathbb{D}(\Xi) 2^{C_{l b}(\Xi)} \prod_{\alpha=1}^{C_{d b}(\Xi)} 2 \cosh \left[2 \mathbf{h}\left(c_{\alpha}^{d b}\right)\right]
$$

where $c_{\alpha}^{d b}$ is the $\alpha$ th dark-blue cluster of $\Xi$ and $\mathbf{h}\left(c_{\alpha}^{d b}\right)$ is defined by

$$
\mathbf{h}\left(c_{\alpha}^{d b}\right)=\beta \sum_{i \in c_{\alpha}^{d b}} h_{i}
$$

We again emphasize that these weights are non-negative and therefore define a probability measure on the space of all RDLB configurations. As above the measures will be denoted by a $\mathbb{V}$; in particular for the Hamiltonian $H$ at inverse temperature $\beta$, in volume $A$ with boundary condition $*$ we will denote these measures by $\mathbb{V}_{A^{*} ; \beta, H}$.

A cluster algorithm based on the RDLB representation is similar to the algorithm described in the previous section. The bond moves are identical. There are now three kinds of spin moves; grey, dark-blue and lightblue. The dark-blue cluster flips occur with probabilities weighted by the exponent of their field energies: For the cluster $c_{\alpha}^{d b}$ the ratio of forward to backward rates is $e^{ \pm 2 \mathbf{h}\left(c_{\alpha}^{d b}\right)}$ with the sign depending on the initial and final spin state. The grey cluster move exchanges light and dark blue cluster with a probability that depends on the fields. Although the grey and dark-blue moves depend on the fields in a complicated way, the light-blue move is independent of the field. The crucial point is that since the field energy is always zero for the light-blue clusters, these are "freely" flipped with probability $1 / 2$. As we demonstrate in the next section, for all ferromagnetic Ising problems, the light-blue infinite cluster density can be identified with the magnetic order parameter. Thus, in this representation, the degrees of freedom contributing to the long range order are decoupled from the fields. Nevertheless, the algorithm may experience slowing due to the presence of large grey or dark-blue clusters. In the final section, we will discuss some strategies that can be used to overcome these shortcomings.

\section{THE ORDER PARAMETER}

In the following we present some elementary results for Ising systems. Although most of these are proved explicitly or implicitly in the standard treatments (e.g., $[\mathrm{G}]$ ) for completeness we will provide a proof sketch. Below we will assume that $\Lambda$ is a finite graph that is a subset of some infinite $\mathbb{L}$ and the Ising Hamiltonian, $\mathscr{H}$ is as given in Eq. (1). We will consider sequences $\left(\Lambda_{k}\right)$ which "tend to $\mathbb{L}$ " meaning $\Lambda_{k+1} \supset \Lambda_{k}$ and each $i \in \mathbb{L}$ 
is, eventually, in some $A_{k}$. This is denoted by $A>\mathbb{L}$. Thermal states on $A$ at inverse temperature $\beta$ and boundary condition \# on $\mathbb{L} \backslash A$ will be denoted by $\langle-\rangle_{A ; \beta, \mathscr{H}}^{\#}$. The special (and most important) cases are $\#=+(\#=-)$ corresponding to each spin of $\mathbb{L} \backslash A$ set to $+(-)$.

Proposition 1. Let $i \in \mathbb{L}$ and for each $A$ with $i \in A$ let

$$
m_{i}^{+}(\Lambda)=\left\langle\sigma_{i}\right\rangle_{\Lambda ; \beta, ~}^{+}
$$

and

$$
m_{i}^{-}(\Lambda)=\left\langle\sigma_{i}\right\rangle_{\Lambda ; \beta, \mathscr{H}}^{-}
$$

Then the limits

$$
m_{i}^{+}=\lim _{\Lambda \rightarrow \mathbb{L}} m_{i}^{+}(A)
$$

and

$$
m_{i}^{-}=\lim _{\Lambda \wedge \mathbb{L}} m_{i}^{-}(\Lambda)
$$

exist for any sequence $\left(A_{k}\right)$ as described above and are independent of the sequence. Let $\mu_{i}(\beta)=\frac{1}{2}\left[m_{i}^{+}(\beta)-m_{i}^{-}(\beta)\right]$ denote the "magnetization excess." Then the necessary and sufficient condition for Gibbsian uniqueness is that for each $i, \mu_{i}(\beta)=0$.

Proof. We use the fact that the finite volume measures (and hence the infinite limits thereof) have the strong FKG property for the ordering induced by $\left(\sigma_{i}=+\right)>\left(\sigma_{i}=-\right)$. This means that the + boundary conditions on $A$ are "higher" than any other boundary conditions. In particular, if $\Lambda^{\prime} \supset \Lambda$ the restriction of $\langle-\rangle_{A^{\prime}, \beta, \mathscr{H}}^{+}$to $A$ is dominated by $\langle-\rangle_{A ; \beta, \mathscr{H}}^{+}$. This ensures the existence of an unambiguous limiting plus state (independent of the sequence $\left(\Lambda_{k}\right)$ ) which will be denoted by $\langle-\rangle_{\beta, \mathscr{H}}^{+}$as well as the existence of the $m_{i}^{+}$. Similar considerations provide the infinite volume state $\langle-\rangle_{\bar{\beta}, \mathscr{H}}$ and the $m_{i}^{-} \equiv\left\langle\sigma_{i}\right\rangle_{\beta, \mathscr{H}}$. If, for any $i, 2 \mu_{i}=m_{i}^{+}-m_{i}^{-}$is not zero then obviously there is more than one state. On the other hand if for every $i, \mu_{i}=0$ then, by Strassen's theorem, [S] we have $\langle-\rangle_{\beta, \mathscr{H}}^{+}=$ $\langle-\rangle_{\beta, \mathscr{H}}$. But since any limiting state lies above $\langle-\rangle_{\beta, \mathscr{H}}$ and below $\langle-\rangle_{\beta, \mathscr{H}}^{+}$(in the sense of $\mathrm{FKG}$ ) it follows that the limiting state is unique. 
Remark. Under the usual physical assumptions that $\mathbb{L}=\mathbb{Z}^{d}$ or some other regular $d$-dimensional lattice and that the $h_{i}$ and $J_{i, j}$ are periodic or i.i.d. random variables then

$$
\mu(\beta)=\lim _{\Lambda>\mathbb{L}} \frac{1}{|\Lambda|} \sum_{i \in A} \mu_{i}(\Lambda) \equiv \lim _{\Lambda>\mathbb{L}} \frac{1}{|A|} \sum_{i \in \Lambda} \frac{1}{2}\left[m_{i}^{+}(\Lambda)-m_{i}^{-}(\Lambda)\right]
$$

exists (almost surely in the random cases) for all $A \nearrow \mathbb{L}$ in the sense of Van Hove. The quantity $\mu(\beta)$ may be identified with a thermodynamic derivative. Indeed if $f(\mathrm{~b})$ is the free energy in the presence of the applied field $\mathfrak{b}$ (that is to say the term $-\sum_{i} \mathrm{~b} \sigma_{i}$ is added to $\mathscr{H}$ ) then $\mu(\beta)=$ $-\frac{1}{2} \beta\left[f^{\prime}\left(\mathbf{b}=0^{+}\right)-f^{\prime}\left(\mathbf{b}=0^{-}\right)\right]$.

Our main result:

Theorem 2. Let $A$ denote a finite graph (which may be regarded as a subset of some infinite $\mathbb{1}$ ) and consider the light-blue wired measure $V_{A^{w} ; \beta, H}(-)$ on RDLB configurations as described in Eqs. (13)-(15) with wired boundary conditions. Let $i \in \Lambda$ and let $\{i \leftrightarrow \partial \Lambda\}$ denote the event that $i$ is connected to the boundary by light-blue bonds. Then

$$
\mathbb{V}_{A^{\mathrm{w}} ; \beta, H}(\{i \leftrightarrow \partial \Lambda\})=\frac{1}{2}\left[m_{i}^{+}(\beta, \Lambda)-m_{i}^{-}(\beta, A)\right] \equiv \mu_{i}(\beta ; A)
$$

Proof. Let $\langle-\rangle{ }_{A ; \beta}^{+},{ }_{B}$ denote the thermal state for the (four-state spin) model on $A$ with Hamiltonian $H$ as in Eq. (13) and +- boundary conditions. It is clear that if $A$ is any function of the $\sigma$ 's alone then $\langle A\rangle_{A ; \beta, H}^{+}=\langle A\rangle_{A ; \beta, \mathscr{H}}^{+}$(because the $\sigma$ 's and the $\tau^{\text {'s }}$ are independent) and similarly if $A^{\prime}$ is a function of the $\tau^{\prime}$ 's alone then $\left\langle A^{\prime}\right\rangle_{A ; \beta}^{+}, \bar{\beta}_{, H}=\left\langle A^{\prime}\right\rangle_{A ; \beta, \mathscr{H}}$. Thus

$$
\frac{1}{2}\left\langle\left[\sigma_{i}-\tau_{i}\right]\right\rangle_{\Lambda ; \beta, H}^{+-}=\frac{1}{2}\left[m_{i}^{+}(\Lambda)-m_{i}^{-}(\Lambda)\right]=\mu_{i}(\Lambda)
$$

The stated identity follows easily from the Edwards-Sokal measure. We start with

$$
\frac{1}{2}\left\langle\left[\sigma_{i}-\tau_{i}\right]\right\rangle_{\Lambda ; \beta, H}^{+-\bar{\beta}, H}=\mathbb{E}_{\Lambda^{\prime w}}^{E S}\left(\frac{1}{2}\left[\sigma_{i}-\tau_{i}\right]\right)
$$

where $\mathbb{E}_{\Lambda^{\text {tw }}}^{E S}(-)$ denotes expectation with respect to the measure $\mathbb{V}_{\Lambda^{\text {tw }}}^{E S}(-)$ with light-blue wired boundary conditions on $\partial A$. Let us consider the three possibilities for the site $i$ : (1) the event $\{i \leftrightarrow \partial \Lambda\}$ occurs, (2) the site $i$ belongs to a dark-blue cluster and (3) the site $i$ belongs to a light-blue cluster that is not connected by light-blue bonds to the boundary. We may express the right hand side of Eq. (17) in terms of the three associated conditional expectations. 
Whenever the third possibility occurs, regardless of whether $i$ is grey connected to $\partial \boldsymbol{A}$, the cluster of $i$ is +- and -+ each with probability $1 / 2$. If $i$ is in a dark-blue cluster then $\sigma_{i}=\tau_{i}$. Thus, items (2) and (3) contribute nothing. On the other hand, if $\{i \leftrightarrow \partial \Lambda\}$ occurs then the boundary conditions force $\sigma_{i}=+$ and $\tau_{i}=-$. This establishes the stated identity.

Remark. It is observed that this representation may also be used for the calculation of correlation functions. For example, consider an Ising system in finite volume $A$ with fields $\left(h_{i}\right)$ and boundary condition $*$. Duplicating the system with the same boundary conditions it is not hard to see that the expectation of $\left(\sigma_{i}-\tau_{i}\right)\left(\sigma_{j}-\tau_{j}\right)$ is exactly twice the truncated spin-spin correlation function between the sites $i$ and $j$. Now if the site $i$ belongs to a dark-blue cluster-which is necessitated if it is connected to the boundary - then the contribution is zero because $\sigma_{i}=\tau_{i}$. Similarly if $j$ belongs to a dark-blue cluster. Furthermore, if $i$ and $j$ belong to separate light-blue clusters there is cancellation because for fixed $\tau_{j}$ and $\sigma_{j}$, the quantity $\left(\sigma_{i}-\tau_{i}\right)$ takes on the value 2 and -2 with equal probability. Finally, if $i$ and $j$ are in the same light-blue cluster, the result is four. Thus we are left with another identity: The truncated spin-spin correlation function is half the probability that the relevant sites are in the same light blue cluster.

With the exception of a few technical points the picture is complete. Given +- (i.e., light-blue wired) boundary conditions, there either is or isn't percolation of light-blue bonds. If there is, the stated order parameter is positive and if not, there is no ordering of the stated type. In fact, these results apply to other systems of this type. For example, the previous sentence holds generally for the Ashkin-Teller model in an external field where $0 \leqslant \mathscr{E}_{i, j}^{1} \leqslant \frac{1}{2} \mathscr{E}_{i, j}^{0}$. However, specific to the Ising system is the stronger statement that if the magnetic excess vanishes $(\mu(\beta) \equiv 0)$, there is no long-range order of any type. There is a second issue that is not unrelated to the first. We have implicitly "defined" percolation to mean percolation in the state that ultimately produces the order parameter. (Of course, in simulations these are rarely the boundary conditions that are actually used.) What has not been ruled out is the possibility that there is light-blue percolation in some limiting state but not in the limiting +- state. Although there can be little doubt that in general this does not occur, for Ising systems we have a complete proof.

We start with a precise definition of (light-blue) percolation:

Definition. Let $A \subset \mathbb{L}$ be a finite lattice and consider the various measures $\mathbb{V}_{A^{*} ; \beta}(-)$ on RDLB configurations with boundary conditions \#. 
For $i \in \Lambda$, consider the probability that $i$ is connected to $\partial \Lambda$ by light-blue bonds maximized over all possible boundary conditions:

$$
P_{i}(\beta ; \Lambda)=\max _{\#} \bigvee_{A^{*} ; \beta, H}(\{i \leftrightarrow \partial A\})
$$

We define

$$
P_{i}(\beta ; \infty)=\lim _{A>\mathbb{L}} P_{i}(\beta ; A)
$$

(It is not hard to show that this limit exists independent of the sequence $\left(\Lambda_{k}\right)$.) If $P_{i}(\beta ; \infty) \neq 0$ for any $i$, we say there is percolation and if $P_{i}(\beta ; \infty) \equiv 0$ there is no percolation.

For Ising systems, we have the following characterization:

Theorem 3. For an Ising system as defined by the Hamiltonian in Eq. (1) there is a unique limiting state if and only if there is no percolation.

Proof. By Proposition 1, there is uniqueness iff $\mu_{i}=0$ for all $i$. Obviously $\mu_{i}(\beta) \leqslant P_{i}(\beta ; \infty)$. It remains to be established that $\mu_{i}(\beta)=$ $0 \Rightarrow P_{i}(\beta ; \infty)=0$. Here an argument similar to that used in [CM II] Theorem 3.8 may be applied. Let $*$ denote any boundary conditions that optimize $\mathbb{V}_{A^{*} ; \beta}(\{i \leftrightarrow \partial \Lambda\})$ which, without loss of generality, is taken to be a single-spin specification. There are (at most) four components to $\partial A$ : $\partial \Lambda^{++}(*), \partial \Lambda^{--}(*)$ and $\partial \Lambda^{ \pm \mp}(*)$ corresponding to the four spin-types. We may write $P_{i}(\beta ; \Lambda)=P_{i}^{+-}(\beta ; \Lambda)+P_{i}^{-+}(\beta ; \Lambda)$ with

$$
P_{i}^{+-}(\beta ; A)=\mathbb{V}_{A^{*} ; \beta, H}\left(\left\{i \leftrightarrow \partial \Lambda^{+-}(*)\right\}\right)
$$

etc. By $\sigma \leftrightarrow \underline{\tau}$ symmetry, it may be assumed that $P_{i}^{+-}(\beta ; A) \geqslant P_{i}^{-+}(\beta ; A)$.

We claim that in the light-blue wired (i.e., +- ) boundary conditions, the probability of $\left\{i \leftrightarrow \partial A^{+-}(*)\right\}$ is at least as large as it is in the $*$ boundary conditions and hence, after the infinite volume limit, $\mu_{i}(\beta) \geqslant \frac{1}{2} P_{i}(\beta ; \infty)$. Let us consider the situation from the perspective of the Edwards-Sokal measure. Decomposing $P_{i}^{+-}(\beta ; A)$ according to spin configurations we have

$\mathbb{V}_{A^{*} ; \beta, H}\left(\left\{i \leftrightarrow \partial A^{+-}(*)\right\}\right)=\sum_{\underline{g}} \mathbb{V}_{A^{*} ; \beta, H}^{E S}(\sigma, \underline{\tau}) \mathbb{V}_{A^{*} ; \beta, H}^{E S}\left\{\left\{i \leftrightarrow \partial A^{+-}(*)\right\} \mid \underline{\sigma}, \underline{\tau}\right)$

The right hand side is of the form of a thermal expectation of a function of spin configurations, namely $\mathbb{V}_{A^{*} ; \beta, H}^{E S}\left(\left\{i \leftrightarrow \partial \Lambda^{++}(*)\right\} \mid \underline{\sigma}, \tau\right)$;

$$
\mathbb{V}_{A^{*} ; \beta, H}\left(\left\{i \leftrightarrow \partial A^{+-}(*)\right\}\right)=\left\langle\mathbb{V}_{A^{*} ; \beta, H}^{E S}\left(\left\{i \leftrightarrow \partial A^{+-}(*)\right\} \mid \underline{\sigma}, \underline{\tau}\right)\right\rangle_{\beta, H ; A}^{*}
$$


However, in light of the algorithmic construction of the configurations $\Xi$ given $\underline{\sigma}$ it is clear that this function is increasing in $\underline{\sigma}$ and decreasing in $\underline{\tau}$ : Indeed, this is amounts to a bond-site percolation problem and increasing the number of plus $\sigma$ 's and minus $\tau$ 's just increases the number of sites. But then, by the FKG property of the Gibbs measures,

$$
\begin{aligned}
& \left\langle\mathbb{V}_{A^{*} ; \beta, H}^{E S}\left(\left\{i \leftrightarrow \partial A^{+-}(*)\right\} \mid \underline{\sigma}, \underline{\tau}\right)\right\rangle_{A ; \beta, H}^{*} \\
& \quad \leqslant\left\langle\mathbb{V}_{A^{w} ; \beta, H}^{E S}\left(\left\{i \leftrightarrow \partial A^{+-}(*)\right\} \mid \underline{\tau}, \underline{\tau}\right)\right\rangle_{A ; \beta, H}^{+-} \\
& \quad=\mathbb{V}_{A^{w} ; \beta, H}\left(\left\{i \leftrightarrow \partial A^{+-}(*)\right\}\right) \leqslant \mu_{i}(\beta ; A)
\end{aligned}
$$

from which the stated result follows.

\section{CONCLUSIONS}

The upshot of the previous analysis is that the crucial degrees of freedom at points where there is a magnetic ordering transition are represented by the light-blue clusters. For the algorithms described, the spin-transitions within these clusters is uninhibited and this should lead to substantial improvement over other cluster methods in many situations. On the other hand, when the system is critical or at a point of phase coexistence, it may be too much to hope that there are never any other large-scale fluctuations. In particular, in the absence of fields, by light/dark symmetry, the distribution of dark-blue clusters is identical to that of the light-blue. In the presence of fields, there might still be large dark-blue clusters and these would have a tendency to "freeze." Furthermore, since we expect grey percolation at all points of physical interest, the transitions that exchange dark with light could be disastrously slow.

However even assuming the worst case scenario, namely that the only aspects worth preserving are the light-blue moves, the algorithm may be supplemented with Monte Carlo steps from other algorithms to insure ergodicity. In these circumstances, the whole of the previously described algorithm need not be implemented. Indeed, the following "pure light-blue" move clearly satisfies detailed balance: Starting from a spin-configuration $\underline{s}$, between all neighboring pairs $\langle i, j\rangle$ where $s_{i}=s_{j}=+-$ or $s_{i}=s_{j}=-+$ independently place a light-blue bond with probability $1-e^{-4 \beta J_{i, j}}$. Clusters are identified and, independently, each cluster is flipped or left alone with probability $\frac{1}{2}$. Such moves (which are obviously not ergodic) can then be sandwiched between single-spin updates.

In addition (for Ising systems) let us recall that it is only the statistical behavior of single layers that is actually of interest. Thus we may consider an ensemble of replicas where, in the cluster moves, members of the 
ensemble are paired randomly. Furthermore, if there is some translation invariance (e.g., the staggered-field problem) then the replicas may be translated relative to one another. Of course neither of the above moves will restore ergodicity; other types of updates are still required.

A closely related set of ideas described in [ $\mathrm{HBb}]$ and $[\mathrm{HBc}]$ has recently been brought to our attention. Here the idea is to "fold" the lattice through an axis of symmetry; e.g., the $x_{1}$-axis. Spins at the sites $i=$ $\left(x_{1}, x_{2}, \ldots, x_{d}\right)$ and $i^{*}=\left(-x_{1}, x_{2}, \ldots, x_{d}\right)$ are now regarded as "pairs." Assuming that $h_{i}=h_{i^{*}}$ (as would be the case for the staggered field problem) one may implement a procedure to generate light-blue clusters as described above. In the references cited, single cluster methods were used. Aside from the "boundary spins," i.e., the $x_{1}$-axis, the method of folding and of duplication are, for all intents and purposes, identical for problems with periodically varying fields. However, the advantage of having two separate replicas is manifestly apparent for systems where $h_{i}$ is random or, more generally, has no spatial periodicity.

Recently, the authors tested some of these strategies on the square lattice staggered-field problem [RMC]. The algorithm was applied to a tworeplica system and consisted of light-blue cluster moves, random translations of one replica relative to the other by even or odd lattice vectors, and Metropolis updates. In the case of odd translations, one replica is also globally flipped relative to the other. In [RMC] we use the terminology "active sites" ("inactive sites") to refer to sites in light-blue (dark-blue) clusters since, in the cluster part of the algorithm, only active sites are flipped. The results were quite satisfactory; for systems up to scale 256 , our data is consistent with a critical slowing exponent less than 0.5 (compared with local dynamics where the value is found to be in excess of 2 ).

\section{ACKNOWLEDGMENTS}

J.M. was partially supported by NSF grant DMR-9632898, L.C. by NSA grant MDA904-98-1-0518.

\section{REFERENCES}

[ACCN] M. Aizenman, J. T. Chayes, L. Chayes, and C. M. Newman, Discontinuity of the magnetization in one-dimensional $1 /|x-y|^{2}$ Ising and Potts models, J. Stat. Phys. 50:1-40 (1988).

[CK] A. Coniglio and W. Klein, Clusters and Ising critical droplets: A renormalization froup approach, J. Phys. A: Math. Gen. 13:2775-2780 (1980).

[CM I] L. Chayes and J. Machta, Graphical representations and cluster algorithms. Part I: Discrete spin systems, Physica A 239:542-601 (1997). 
[CM II] L. Chayes and J. Machta, Graphical representations and cluster algorithms. Part II, Physica A 254:477-516 (1998).

[ES] R. G. Edwards and A. D. Sokal, Generalization of the Fortuin-KasteleynSwendsen-Wang representation and Monte Carlo algorithm, Phys. Rev. D 38: 2009-2012 (1988).

[FK] C. M. Fortuin and P. W. Kasteleyn, On the random cluster model. I. Introduction and relation to other models, Physica 57:536-564 (1972).

[G] H. O. Georgii, Gibbs Measures and Phase Transitions (Walter de Gruyter, Berlin, 1988).

[HBa] J. R. Heringa and H. W. J. Blöte, The simple cubic lattice gas with nearest neighbor exclusion: Ising universality, Physica A 232:369-374 (1996).

[HBb] J. R. Heringa and H. W. J. Blöte, Geometric cluster Monte Carlo simulation, Phys. Rev. E 57:4976-4978 (1998).

[ $\mathrm{HBc}] \quad$ J. R. Heringa and H. W. J. Blöte, Cluster dynamics and universality of Ising lattice gases, Preprint (1998).

[KD] D. Kandel and E. Domany, General cluster Monte Carlo dynamics, Phys. Rev. $B$ 43:8539-8548 (1991).

[NB] M. E. J. Newman and G. T. Barkema, Monte Carlo study of the random-field Ising model, Phys. Rev. E 53:393-403 (1996).

[RMC] O. Redner, J. Machta, and L. F. Chayes, Graphical representations and cluster algorithms for critical points with fields, Phys. Rev. E 58:2749-2752 (1998) and cond-mat $/ 9802063$.

[S] Strassen, The existence of probability measures with given marginals, Ann. Math. Statist. 36:423-439 (1965).

[SW] R. H. Swendsen and J. S. Wang, Nonuniversal critical dynamics in Monte Carlo simulations, Phys. Rev. Lett. 58:86 (1987). 